\title{
Erratum zu: Die Sterbehilfedebatte und das Bild der Palliativmedizin in deutschen Printmedien
}

\author{
Marie-Christin Hahnen • Tania Pastrana • Stephanie Stiel • \\ Arnd May • Dominik Groß $\cdot$ Lukas Radbruch
}

Online publiziert: 5. November 2009

(C) Springer-Verlag 2009

\section{Erratum zu: Ethik Med (2009) \\ DOI 10.1007/s00481-009-0033-8}

In der ursprünglichen Fassung des Artikels wurde in der Diskussion ein Satz nicht richtig wiedergegeben. Der korrekte Satz lautet: Einerseits sind Depressionen behandelbar und andererseits neigen gerade depressive Patienten dazu, ihre Einstellung zur Sterbehilfe oder zum assistierten Suizid zu ändern [18].

Die Onlineversion des Originalbeitrages ist erreichbar unter doi:10.1007/s00481-009-0033-8

M.-C. Hahnen $(\bowtie)$

Klinik für Palliativmedizin, Universitätsklinikum Aachen, Pauwelsstr. 30 52074 Aachen, Deutschland

E-Mail: marie.hahnen@rwth-aachen.de

T. Pastrana $\cdot$ S. Stiel $\cdot$ L. Radbruch

Klinik für Palliativmedizin, Universitätsklinikum Aachen, Aachen, Deutschland

A. May · D. Groß

Institut für Geschichte, Theorie und Ethik in der Medizin, RWTH Aachen, Aachen, Deutschland 